\title{
Gonorrhea in Canada: 2003-2012
}

\author{
Totten $\mathbf{S}^{1, *}$, MacLean $\mathbf{R}^{1}$, Payne $\mathrm{E}^{1}$ \\ ${ }^{1}$ Centre for Communicable Diseases and Infection Control, Public Health Agency of Canada, Ottawa, ON \\ ${ }^{*}$ Correspondence: Stephanie.Totten@phac-aspc.gc.ca
}

\section{Abstract}

Background: Gonorrhea is the second most commonly reported sexually transmitted infection in Canada. Between 1991 and 1997, Canada experienced a sharp decline in the rates of reported cases of gonorrhea, followed by a steady incline.

Objective: To identify trends in reported cases of gonorrhea in Canada from January 1, 2003 to December 31 , 2012.

Methods: Notifiable disease reports were submitted to the Public Health Agency of Canada by provincial and territorial epidemiological units and data were summarized by age and sex.

Results: Between 2003 and 2012, the rate of reported cases of gonorrhea increased by $38.9 \%$, from 26.0 to 36.2 per 100,000. Over this time frame, a greater relative rate increase was observed in females, though rates of gonorrhea increased in both sexes and across all age groups. In 2012, as in previous years, the rate of reported cases of gonorrhea was higher in males than females (41.4 vs. 31.0 per 100,000). Females between the ages of 15 and 24 years and males between the ages of 20 and 29 years accounted for the highest rates of gonorrhea in 2012.

Conclusion: In Canada, as in many countries, gonorrhea is on the rise, especially in young adults. This increase in rates of reported cases is in part due to improved diagnosis through nucleic acid amplification (NAAT) testing and may also be affected by growing gonococcal resistance to many available treatments.

\section{Introduction}

Gonorrhea has been nationally notifiable since 1924 and is the second most commonly reported sexuallytransmitted infection (STI) in Canada. Gonorrhea is a genital bacterial infection caused by Neisseria gonorrhoeae and if left untreated, can lead to complications for both sexes. There can be severe consequences for females, including pelvic inflammatory disease, which often leads to chronic abdominal pain, infertility and ectopic pregnancy. In males, untreated infections can result in epididymitis and rare cases of infertility. An uncommon complication of gonorrhea is the spread of infection to the blood stream and joints (1). Like other STIs, gonorrhea increases the risk of HIV acquisition and transmission, possibly by increasing the concentration of HIV target cells in genital secretions and viral shedding (2).

Between 1991 and 1997, Canada experienced a sharp decline in the rates of reported cases of gonorrhea and rates were similar between males and females. After 1997, rates began to rise (3).

The objective of this report is to summarize the trends in gonorrhea rates in Canada between 2003 and 2012. It is based on the Report on Sexually Transmitted Infections in Canada: 2012 prepared by the Centre for Communicable Diseases and Infection Control of the Public Health Agency of Canada (PHAC) (available online) (4). 


\section{Methods}

\section{Data collection}

Data on laboratory-confirmed gonorrhea cases were reported to the Canadian Notifiable Disease Surveillance System (CNDSS) by provincial and territorial health authorities according to the Agency's Case Definitions for Communicable Diseases under National Surveillance (5) (see box below). Data are submitted in a variety of formats (e.g., line-listed electronic, paper-based case reports, or aggregate data) and are verified and loaded into the CNDSS database by Agency personnel.

\section{Case definitions of confirmed case of gonorrhea}

Laboratory-evidence of infection in genitourinary or extra-genital specimens:

- Detection of Neisseria gonorrhoeae by culture,

OR

- Detection of N. gonorrhoeae nucleic acid.

\section{Data analysis}

Gonorrhea data extracted from the CNDSS were analyzed by Centre for Communicable Diseases and Infection Control (CCDIC) staff. Rates were calculated by age group and sex using population estimates obtained from the Statistics Canada Demography Division. Rate change calculations were made on unrounded figures for greater precision. Surveillance is considered to be within the mandate of Federal/Provincial/Territorial (F/P/T) programs and does not constitute human research; therefore, no research ethics board approval was sought.

\section{Results}

In 2012, 12,561 cases of gonorrhea were reported through the CNDSS, corresponding to a rate of 36.2 per 100,000 . The 2012 rate was a $38.9 \%$ increase from the rate of 26.0 per 100,000 in 2003 . Over this ten-year time frame, rates increased among both males and females; males experienced a $29.1 \%$ relative rate increase while females experienced a $53.9 \%$ relative rate increase. Around 2006, rates of reported cases of gonorrhea began to show signs of stabilizing, with minor fluctuations from year to year. (Figure 1).

Figure 1: Overall and sex-specific rates of gonorrhea, 2003 to 2012, Canada

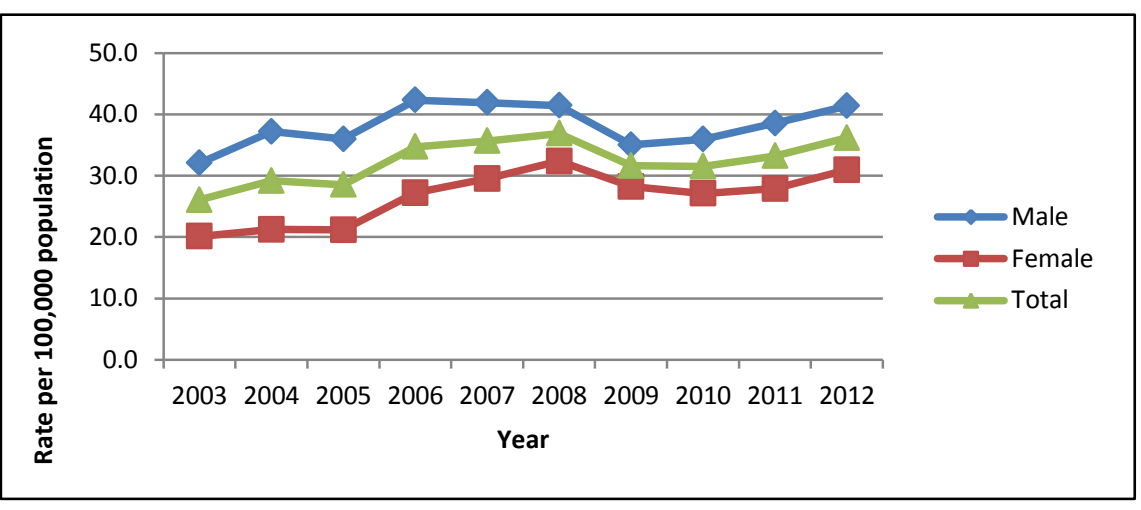

Rates of reported cases of gonorrhea in 2012 were higher among females than males in those less than 25 years of age; but were higher in males than females among those aged 25 years and over (Figure 2). The highest rates of gonorrhea were observed among males and females aged 20 to 24 .

Between 2003 and 2012, rates of reported cases of gonorrhea increased among both males and females aged 10 and above. The greatest relative rate increase observed among males was in those aged 10 to 14 years 
(262.0\%), from 0.5 to 1.7 per 100,000 . Over this ten-year time frame, the highest relative increase observed among females was in those aged 60 and over $(188.0 \%$, from 0.2 to 0.7 per 100,000$)$, though females in this age group exhibited the lowest rate of gonorrhea as compared to females in other age groups (data not shown).

Figure 2: Rates of reported gonorrhea by sex and age group, 2012, Canada

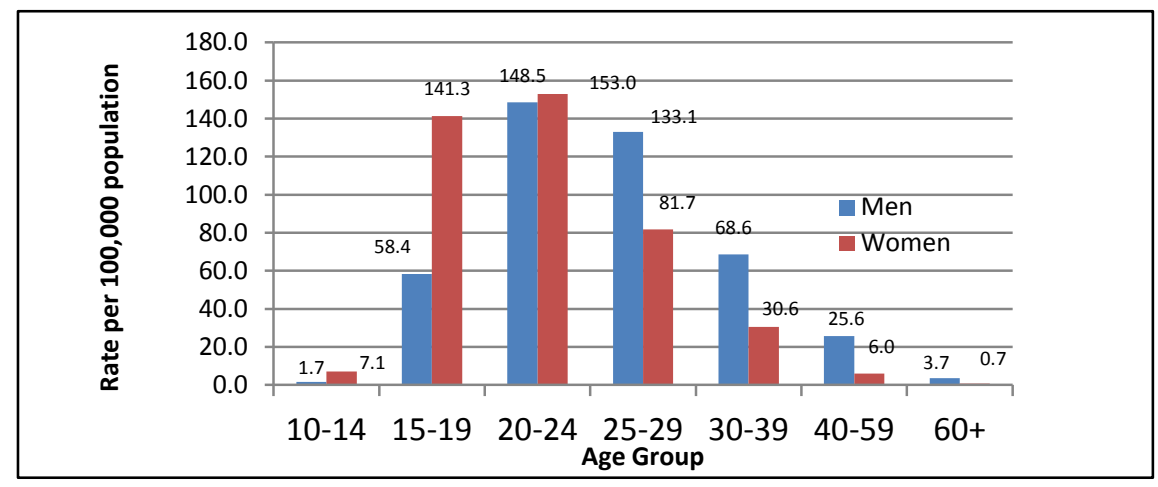

Between 2003 and 2012, most provinces and territories experienced a relative increase in the rate of the reported cases of gonorrhea (data not shown).

\section{Discussion}

Recent trends in the rates of reported cases of gonorrhea in Canada have shown overall increases, despite some year-to-year variation. The increases in rates since the late 1990 s may be at least partly explained more sensitive testing methods and improved case finding (6).

Antimicrobial resistance may also play a significant role in the increase in reported rates of gonorrhea, as the proportion of isolates resistant to a number of antibiotics has increased over time, (7) which may lead to treatment failure and a longer duration of infectiousness in affected patients. Emerging antimicrobial resistance in gonorrhea has led to changes in treatment recommendations across Canada and elsewhere $(8,9,10,11)$.

In 2012, the overall rates of reported cases of gonorrhea were substantially lower in Canada at 36.2 per 100,000 compared to the United States (107.5 per 100,000) (12), Australia (58.9 per 100,000) (13) and England (48.1 per $100,000)(14)$. There was considerable variability in the differences observed across sexes; in Australia and England, gonorrhea rates were more than twice as high among males as compared to females, while the differences between sexes in Canada and the United States were less pronounced.

In conclusion, over a 10 -year period, gonorrhea rates have increased almost $40 \%$ and these increases are particularly pronounced among young adults. While the reasons for these increases are likely multifactorial, it is possible that increasing gonococcal resistance to many available treatments has contributed to observed trends and should continue to be monitored.

\section{Acknowledgements}

The full report was prepared by the Centre for Communicable Diseases and Infection Control, Infectious Disease Prevention and Control Branch, Public Health Agency of Canada. Its publication would not have been possible without the collaboration of all provinces and territories, whose continuous contribution to national STI surveillance is greatly appreciated. The authors also acknowledge the contributions and expertise of the Sexually Transmitted and Blood-Borne Infections Surveillance Network.

\section{Conflict of interest}

None 


\section{Funding}

This work was supported by the Public Health Agency of Canada.

\section{References}

(1) Hook EW, Handsfield HH. Gonococcal infections in the adult. In: Holmes KK, Sparling PF, Mardh P, Lemon SM, Stamm WE, Piot P, et al., editors. Sexually transmitted diseases. 3rd ed. New York: Mc-Graw Hill; 1999. p. 451-466.

(2) Fleming DT, Wasserheit JN. From epidemiological synergy to public health policy and practice: The contribution of other sexually transmitted diseases to sexual transmission of HIV infection. Sex Transm Infect. 1999 Feb;75(1):3-17.

(3) Public Health Agency of Canada. Report on sexually transmitted infections in Canada: 2011. Centre for Communicable Diseases and Infection Control, Infectious Disease Prevention and Control Branch, Public Health Agency of Canada; 2014. 2011. http://publications.gc.ca/site/eng/469949/publication.html.

(4) Public Health Agency of Canada. Report on sexually transmitted infections in Canada: 2012. Centre for Communicable Diseases and Infection Control, Infectious Disease Prevention and Control Branch, Public Health Agency of Canada; 2015.

(5) Public Health Agency of Canada. Case definitions for communicable diseases under national surveillance Can Comm Dis Report. 2009; 35:S-2.

(6) Rekart ML, Brunham RC. Epidemiology of chlamydial infection: are we losing ground? Sex Transm Infect. 2008 Apr;84(2):87-91.

(7) Martin I, Sawatzky P, Liu G, Mulvey MR. Antimicrobial resistance to Neisseria gonorrhoeae in Canada: 2009-2013. CCDR. 2015 Feb;41(2).

(8) Plitt S, Boyington C, Sutherland K, Lovgren M, Tilley P, Read R, et al. Antimicrobial resistance in gonorrhea: The influence of epidemiologic and laboratory surveillance data on treatment guidelines: Alberta, Canada 2001-2007. Sex Transm Dis. 2009 Oct;36(10):665-669.

(9) Public Health Agency of Canada. Important notice: Public health information update on the treatment for gonococcal infection. 2011. http://www.phac-aspc.gc.ca/std-mts/sti-its/alert/2011/alert-gono-eng.php.

(10) Ontario Agency for Health Protection and Promotion (Public Health Ontario). Guidelines for testing and treatment of gonorrhea in Ontario. 2013.

(11) Centers for Disease Control and Prevention. Update to CDC's sexually transmitted diseases treatment guidelines, 2010: Oral cephalosporins no longer a recommended treatment for gonococcal infections. Morb Mortal Wkly Rep. 2012;61(31):590-594.

(12) Centers for Disease Control and Prevention. Sexually transmitted disease surveillance 2012. Atlanta: U.S. Department of Health and Human Services; 2013. http://www.cdc.gov/std/stats12/.

(13) The Kirby Institute. HIV, viral hepatitis and sexually transmissible infections. In Australia annual surveillance report 2013. The Kirby Institute, The University of New South Wales, Sydney NSW 2052. https://kirby.unsw.edu.au/surveillance/2013annual-surveillance-report-hiv-viral-hepatitis-stis.

(14) Public Health England. Table 1: Number \& rates of new STI diagnoses in England, 2003 - 2012. https://www.gov.uk/government/statistics. 